\title{
A Job-Shop Scheduling Problem with Fuzzy Processing Times
}

\author{
Feng-Tse Lin \\ Department of Applied Mathematics, Chinese Culture University \\ YangminShan, Taipei, Taiwan, ROC \\ ftlin@staff.pccu.edu.tw
}

\begin{abstract}
Job-shop scheduling is a difficult problem, both theoretically and practically. This problem is a combinatorial optimization of considerable industrial importance. Although the job-shop scheduling problem has often been investigated, very little of this research is concerned with the uncertainty characterized by the imprecision in problem variables. In this paper, we investigate the job-shop scheduling problem with imprecise processing times. We first use triangular fuzzy numbers to represent imprecise processing times, and then construct a fuzzy job-shop scheduling model. Our work intends to extend the deterministic job-shop scheduling problem into a more generalized problem that would be useful in practical situations.
\end{abstract}

\section{Introduction}

The job-shop scheduling problem is concerned with allocating limited resources to operations over time [3]. Between the operations precedence constraints for a job can be defined. Although job-shop scheduling has always had an important role in the field of production and operations management, it is a difficult problem in combinatorial optimization [12]. The difficulty is due to the high number of constraints, unfortunately unavoidable in the real-world applications [9]. The job-shop scheduling problem can be described as follows. We are given $n$ jobs and $m$ machines. Each job consists of a sequence of operations that must be processed on $m$ machines in a given order. Each operation must be executed uninterrupted on a given machine for a given period of time and each machine can only handle at most one operation at a time. The problem is to find a schedule, an allocation of the operations of $n$ jobs to certain time intervals on $m$ machines, with a minimum overall time.

Solving the job-shop scheduling problem requires a high computational effort and considerable sophistication [2, 4]. Instead of investigating using optimal algorithms, it is often preferred to use approximation algorithms such as heuristics and meta-heuristics, e.g. simulated annealing, genetic algorithms, and tabu search [1, $5,6-9,15,17,19]$. However, most of the methods proposed in the literature required the assumption that all time parameters are known exactly. This is a strong assumption, which may cause severe difficulties in practice. An example is the difficulty in estimating the exact processing times for all jobs on the machines. In fact, there are many vaguely formulated relations and imprecisely quantified physical data 
values in real world descriptions since precise details are simply not known in advance. Stochastic methods exist, but not many address imprecise uncertainty. Although the job-shop scheduling problem has often been investigated, very few of these studies take uncertainty, typified by the imprecision or vagueness in time estimates, into account $[10,14,15,16,18]$.

In this study, we investigate a job-shop scheduling problem with imprecise processing time and use fuzzy numbers to represent imprecise processing times in this problem. The main interest of our approach is that the fuzzy schedules obtained from Property 1 are the same type as those in the crisp job-shop scheduling problem. The fuzzy job-shop scheduling model, in the case of imprecise processing times, is then an extension of the crisp problem.

\section{Job-Shop Scheduling Problem}

The deterministic job-shop scheduling problem is stated as follows. There are $n$ jobs to be scheduled on $m$ machines. Each job consists of a sequence of operations that must be processed on $m$ machines in a given order [13]. Each operation is characterized by specifying both the required machine and the fixed processing time. Several constraints on jobs and machines, which are listed as follows [11]:

(1) Each job must pass through each machine once and only once.

(2) Each job should be processed through the machine in a particular order.

(3) Each operation must be executed uninterrupted on a given machine.

(4) Each machine can only handle at most one operation at a time.

The problem is to find a schedule to determine the operation sequences on the machines in order to minimize the total completion time. Let $c_{i k}$ denote the completion time of job $i$ on machine $k$, and $t_{i k}$ denote the processing time of job $i$ on machine $k$. For a job $i$, if the processing on machine $h$ precedes that on machine $k$, we need the following constraint:

$$
c_{i k}-t_{i k} \geq c_{i h}
$$

On the other hand, if the processing on machine $k$ comes first, the constraint becomes

$$
c_{i h}-t_{i h} \geq c_{i k}
$$

Thus, we need to define an indicator variable $x_{i h k}$ as follows:

$$
x_{i h k}=\left\{\begin{array}{l}
1, \text { processing on machine } h \text { precedes that on machine } k \text { for job } i \\
0, \text { otherwise }
\end{array}\right.
$$


We can then rewrite the above constraints as follows:

$$
c_{i k}-t_{i k}+L\left(1-x_{i h k}\right) \geq c_{i h}, i=1,2, \ldots, n, h, k=1,2, \ldots, m
$$

where $L$ is a large positive number. Consider two jobs, $i$ and $j$, that are to be processed on machine $k$. If job $i$ comes before job $j$, we need the following constraint:

$$
c_{j k}-c_{i k} \geq t_{j k}
$$

Otherwise, if job $j$ comes first, the constraint becomes

$$
c_{i k}-c_{j k} \geq t_{i k}
$$

Therefore, we also need to define another indicator variable $y_{i j k}$ as follows.

$$
y_{i j k}=\left\{\begin{array}{l}
1, \text { if job } i \text { precedes job } j \text { on machine } k \\
0, \text { otherwise }
\end{array}\right.
$$

We can then rewrite the above constraints as follows:

$$
c_{j k}-c_{i k}+L\left(1-y_{i j k}\right) \geq t_{j k}, i, j=1,2, \ldots, n, k=1,2, \ldots, m
$$

The job-shop scheduling problem with a makespan objective can be formulated as follows:

$$
\begin{array}{ll}
\min \max _{1 \leq \mathrm{k} \leq \mathrm{m}}\left[\max _{1 \leq i \leq n}\left[c_{i k}\right]\right] \\
\text { s.t. } c_{i k}-t_{i k}+L\left(1-x_{i h k}\right) \geq c_{i h}, i=1,2, \ldots, n, h, k=1,2, \ldots, m \\
\quad c_{j k}-c_{i k}+L\left(1-y_{i j k}\right) \geq t_{j k}, i, j=1,2, \ldots, n, k=1,2, \ldots, m \\
c_{i k} \geq 0, i=1,2, \ldots, n, k=1,2, \ldots, m \\
x_{i h k}=0 \text { or } 1, i=1,2, \ldots, n, h, k=1,2, \ldots, m \\
y_{i j k}=0 \text { or } 1, i, j=1,2, \ldots, n, k=1,2, \ldots, m
\end{array}
$$

\section{Constructing a Fuzzy Job-Shop Scheduling Model}

\subsection{Imprecise Processing Time}

As in real life situations, some unexpected events may occur, resulting in small changes to the processing time of each job. Therefore, in many situations, the 
processing time can only be estimated as being within a certain interval. Let us illustrate this point using one example. The example taken from [10] is an industrial case in a chemical environment. The chemical reaction depends not only on the pressure and the temperature but also on the quality of the components. It is very difficult to control this dependence. Therefore, the uncertainties of those reaction times must also be modeled. Because of this interval estimation feature, the representation of processing time for a job can be more realistically and naturally achieved through the use of a fuzzy number. As a result, decision-makers (DM) do not need to give a single precise number to represent the processing time of a job. We use $t_{j k}$, the processing time for job $j$ on machine $k$, to denote the process time for each job in this paper. However, $t_{j k}$ is just an estimate and its exact value is actually unknown.

\subsection{A Fuzzy Job-Shop Sequencing Model}

Consider the schedule for the job-shop problem is performed several times in practical situations. Obviously, the processing time for this schedule at different execution times is not necessarily the same. Therefore, an estimated processing time interval, i.e. $\left[t_{j k}-\Delta_{j k 1}, t_{j k}+\Delta_{j k 2}\right]$, should be given to represent the possible range of values for the processing time. Thus the use of interval $\left[t_{j k}-\Delta_{j k 1}, t_{j k}+\Delta_{j k 2}\right]$ is more appropriate than the use of a single estimate, $t_{j k}$, in practical situations. The DM should carefully determine the parameters $\Delta_{j k 1}$ and $\Delta_{j k 2}$, which satisfy $0<\Delta_{j k 1}<t_{j k}$ and $0<\Delta_{j k 2}$, for defining an acceptable processing time range for any particular problem. After that, the DM can choose an appropriate value from the interval $\left[t_{j k}-\Delta_{j k 1}, t_{j k}+\Delta_{j k 2}\right]$ as an estimate for the processing time for job $j$ on machine $k$. Obviously, when the estimate happens to be $t_{j k}$, which is the crisp processing time, the error rate is zero. When the estimate deviates from $t_{j k}$, the error rate will become larger. In fact, we can use the term "confidence level" instead of "error rate" while we consider processing time interval based on the fuzzy viewpoint. We can therefore say that the confidence level is one if the processing time estimate equals $t_{j k}$. Otherwise, when the processing time estimate deviates from $t_{j k}$, the confidence level will become smaller. Finally, when the estimate approaches one of the two ends of the interval, i.e. $t_{j k}-\Delta_{j k 1}$ or $t_{j k}+\Delta_{j k 2}$, the confidence level will be close to zero.

A level 1 triangular fuzzy number corresponding to the above interval $\left[t_{j k}-\right.$ $\left.\Delta_{j k 1}, t_{j k}+\Delta_{j k 2}\right]$ is given as follows:

$$
\begin{aligned}
& \tilde{t}_{j k}=\left(t_{j k}-\Delta_{j k 1}, t_{j k}, t_{j k}+\Delta_{j k 2} ; 1\right) \in F_{N}(1), \\
& 0<\Delta_{j k 1}<t_{j k}, 0<\Delta_{j k 2}, j=1,2, \ldots, n, k=1,2, \ldots, m .
\end{aligned}
$$




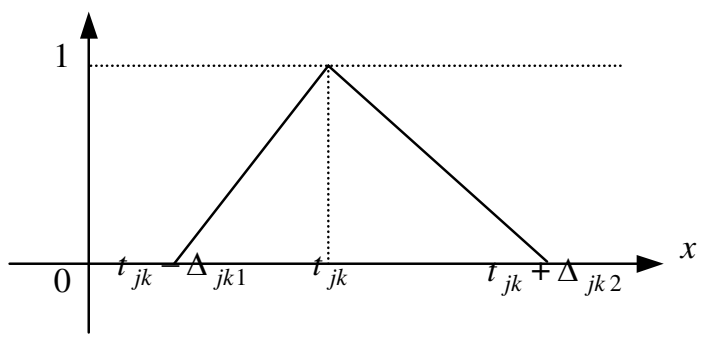

Fig. 1. A triangular fuzzy number $\tilde{t}_{j k}$.

Fig. 1 shows a level 1 triangular fuzzy number $\tilde{t}_{j k}$. From fig. 1 we can see that the membership grade at $t_{j k}$ in $\tilde{t}_{j k}$ is 1 . However, the more the fuzzy number deviates from the $t_{j k}$ position, the lesser the fuzzy numbers membership grade in $\tilde{t}_{j k}$. The membership grade at $t_{j k}-\Delta_{j k 1}$ or $t_{j k}+\Delta_{j k 2}$ is zero. Of course, we can see that the confidence level for an estimate in the interval corresponds to the membership grade of a fuzzy number in the fuzzy sets. This concept naturally leads to the use of fuzzy numbers for the processing time in the job-shop scheduling problem.

Let $\tilde{c}_{i k}=\left(c_{i k}, c_{i k}, c_{i k} ; 1\right)=\left(\tilde{c}_{i k}\right)_{1} \in F_{N}(1)$.

The signed distance of level $\lambda$ fuzzy number is defined as follows. For each $\lambda \in[0,1]$ and $\tilde{A}=(a, b, c ; \lambda) \in F_{N}(\lambda)$. The signed distance of $\tilde{A}$ measured from $\tilde{0}_{1}$ is defined by $d\left(\tilde{A}, \tilde{0}_{1}\right)=\frac{1}{4}(2 b+a+c)$. Then $d\left(\tilde{c}_{i k}, \tilde{0}_{1}\right)=c_{i k}$ is the signed distance from $\tilde{0}_{1}$ to $\tilde{c}_{i k}$. Since $d\left(\tilde{c}_{i k}, \tilde{0}_{1}\right)=c_{i k}>0$, it is a positive distance from $\tilde{0}_{1}$ to $\tilde{c}_{i k}$. Therefore $c_{i k}=d\left(\tilde{c}_{i k}, \tilde{0}_{1}\right)$ is the completion time measured from the origin 0 .

The relations $\succ, \approx$ are the ranking as defined on $F_{N}(1)$. We then fuzzify (1) - (4) using (6) and (7) to obtain the following fuzzy job-shop scheduling problem:

$$
\begin{array}{ll} 
& \min \max _{1 \leq \mathrm{k} \leq \mathrm{m}}\left[\max _{1 \leq \mathrm{i} \leq \mathrm{n}}\left[d\left(\tilde{c}_{i k}, \tilde{0}_{1}\right)\right]\right] \\
\text { s.t. } & \tilde{c}_{i k} \ominus \tilde{t}_{i k} \oplus\left(L\left(\tilde{1}-x_{i h k}\right)\right)_{1} \quad \tilde{c}_{i h}, i=1,2, \ldots, n, h, k=1,2, \ldots, m \\
& \tilde{c}_{j k} \ominus \tilde{c}_{i k} \oplus\left(L\left(\tilde{1}-y_{i j k}\right)\right)_{1} \quad \tilde{t}_{j k}, i, j=1,2, \ldots, n, k=1,2, \ldots, m \\
& \tilde{c}_{i k} \quad \tilde{0}_{1}, i=1,2, \ldots, n, k=1,2, \ldots, m \\
& x_{i h k}=0 \text { or } 1, i=1,2, \ldots, n, k=1,2, \ldots, m \\
& y_{i j k}=0 \text { or } 1, i, j=1,2, \ldots, n, k=1,2, \ldots, m
\end{array}
$$


Note that $\left(L\left(\tilde{1}-x_{i h k}\right)\right)_{1}$ is a fuzzy point at $L\left(1-x_{i h k}\right)$.

Since $d\left(\tilde{c}_{i k}, \tilde{0}_{1}\right)=c_{i k}$,

and $\quad d\left(\tilde{t}_{j k}, \tilde{0}_{1}\right)=t_{j k}+\frac{1}{4}\left(\Delta_{j k 2}-\Delta_{j k 1}\right)$, let $t_{j k}^{*}=d\left(\tilde{t}_{j k}, \tilde{0}_{1}\right)$.

We summarize (9)-(14) to obtain the following Property 1.

Property 1. The job-shop scheduling problem with imprecise processing time modeled by fuzzy number is as follows:

$$
\begin{array}{ll} 
& \min \max _{1 \leq \mathrm{k} \leq \mathrm{m}}\left[\max _{1 \leq i \leq n}\left[c_{i k}\right]\right] \\
\text { s.t. } & c_{i k}-t_{i k}^{*}+L\left(1-x_{i h k}\right) \geq c_{i h}, i=1,2, \ldots, n, h, k=1,2, \ldots, m \\
& c_{j k}-c_{i k}+L\left(1-y_{i j k}\right) \geq t_{j k}^{*}, i, j=1,2, \ldots, n, k=1,2, \ldots, m \\
& c_{i k} \geq 0, i=1,2, \ldots, n, k=1,2, \ldots, m \\
& x_{i h k}=0 \text { or } 1, i=1,2, \ldots, n, h, k=1,2, \ldots, m \\
& y_{i j k}=0 \text { or } 1, i, j=1,2, \ldots, n, k=1,2, \ldots, m
\end{array}
$$

\section{Computational Results}

Consider an example from [11]. We compare the results obtained from Property 1 with that of the crisp job-shop scheduling problem using the Johnson's constructive algorithm. Johnson's algorithm for, $n / 2 / G / F_{\max }$ (i.e. $n$ jobs, two machines, general job-shop problem, to minimize makespan) problem is stated briefly as follows:

Suppose that the set of $n$ jobs $\left\{J_{1}, J_{2}, \ldots, J_{n}\right\}$ may be partitioned into four types of jobs.

Type A: Those to be processed on machine $M_{1}$.

Type B: Those to be processed on machine $M_{2}$.

Type C: Those to be processed on both machines in the order $M_{1}$ then $M_{2}$.

Type D: Those to be processed on the machine in the order $M_{2}$ then $M_{1}$.

Then the construction of an optimal schedule is straightforward, as follows:

(1) Schedule the jobs of type A in any order to give the sequence $S_{A}$.

(2) Schedule the jobs of type B in any order to give the sequence $S_{B}$.

(3) Schedule the jobs of type $C$ according to Johnson's algorithm for $n / 2 / F / F_{\max }$ ( $n$ jobs, 2 machines, flow-shop problem, to minimize makespan) problems to give the sequence $S_{C}$.

(4) Schedule the jobs of type D according to Johnson's algorithm for $n / 2 / F / F_{\max }$ problems to give the sequence $S_{D}$. 
The example is the $9 / 2 / G / F_{\max }$, nine jobs and two machines, with times and processing order as given in Table 1.

Table 1. Processing order and times.

\begin{tabular}{|c|c|c|}
\hline Job & First Machine & Second Machine \\
\hline 1 & $M_{1} 8\left(=t_{11}\right)$ & $M_{2} 2\left(=t_{12}\right)$ \\
2 & $M_{1} 7\left(=t_{21}\right)$ & $M_{2} 5\left(=t_{22}\right)$ \\
3 & $M_{1} 9\left(=t_{31}\right)$ & $M_{2} 8\left(=t_{32}\right)$ \\
4 & $M_{1} 4\left(=t_{41}\right)$ & $M_{2} 7\left(=t_{42}\right)$ \\
5 & $M_{2} 6\left(=t_{52}\right)$ & $M_{1} 4\left(=t_{51}\right)$ \\
6 & $M_{2} 5\left(=t_{62}\right)$ & $M_{1} 3\left(=t_{61}\right)$ \\
7 & $M_{1} 9\left(=t_{71}\right)$ & - \\
8 & $M_{2} 1\left(=t_{82}\right)$ & - \\
9 & $M_{2} 5\left(=t_{92}\right)$ & - \\
\hline
\end{tabular}

An optimal crisp sequence for Table 1, obtained by Johnson's algorithm is

\begin{tabular}{ll} 
& Processing Sequence of jobs \\
\hline Machine $M_{1}$ & $(4,3,2,1,7,5,6)$ \\
Machine $M_{2}$ & $(5,6,8,9,4,3,2,1)$
\end{tabular}

We can see that the total time $F_{\max }=44$ for the optimal sequence. From Property 1 , we consider the following fuzzification for processing time $t_{j k}$.

$\tilde{t}_{11}=(8-2,8,8+4), \tilde{t}_{21}=(7-1,7,7+4), \tilde{t}_{31}=(9-3,9,9+1), \tilde{t}_{41}=(4-1,4,4+2)$,

$\tilde{t}_{51}=(4-0.5,4,4+1), \tilde{t}_{61}=(3-0.6,3,3+1.5), \tilde{t}_{71}=(9-3,9,9+2)$,

$\tilde{t}_{12}=(2-0.8,2,2+1), \tilde{t}_{22}=(5-1,5,5+2), \tilde{t}_{32}=(8-1,8,8+4), \tilde{t}_{42}=(7-2,7,7+3)$,

$\tilde{t}_{52}=(6-2,6,6+1), \tilde{t}_{62}=(5-0.9,5,5+2), \tilde{t}_{82}=(1-0.5,1,1+0.6), \tilde{t}_{92}=(5-2,5,5+1)$

By (14) we obtain the following fuzzy processing times.

$t_{11}^{*}=8.5, t_{21}^{*}=7.75, t_{31}^{*}=8.5, t_{41}^{*}=4.25, t_{51}^{*}=4.125, t_{61}^{*}=3.225, t_{71}^{*}=8.75, t_{12}^{*}=2.05$,

$t_{22}^{*}=5.25, t_{32}^{*}=8.75, t_{42}^{*}=7.25, t_{52}^{*}=5.75, t_{62}^{*}=5.275, t_{82}^{*}=1.025, t_{92}^{*}=4.75$. 
Then we find an optimal schedule as follows:

Type A jobs: job 7 on $M_{1}$.

Type B jobs: jobs 8 and 9 on $M_{2}$ in an arbitrary order $(8,9)$.

Type C jobs: jobs $1,2,3$, and 4 require $M_{1}$ first and then $M_{2}$. Using Johnson's algorithm we obtain the relations $t_{31}^{*}<t_{32}^{*}, t_{41}^{*}<t_{42}^{*}$, and $t_{41}^{*}<t_{31}^{*} ; t_{11}^{*}>t_{12}^{*}$, $t_{21}^{*}>t_{22}^{*}$, and $t_{12}^{*}<t_{22}^{*}$. Thus the sequence obviously is $(4,3,2,1)$.

Type D jobs: jobs 5 and 6 require $M_{2}$ first and then $M_{1}$. Again, using Johnson's algorithm we obtain the relations $t_{51}^{*}<t_{52}^{*}, t_{61}^{*}<t_{62}^{*}$, and $t_{61}^{*}<t_{51}^{*}$. Note that $M_{1}$ now becomes the second machine. The sequence is therefore $(5,6)$. Finally, an optimal sequence for Property 1 is

\begin{tabular}{cc} 
& Processing Sequence of Jobs \\
\hline Machine $M_{1}$ & $(4,3,2,1,7,5,6)$ \\
Machine $M_{2}$ & $(5,6,8,9,4,3,2,1)$ \\
\end{tabular}

The processing sequence for jobs obtained from the fuzzy case is the same as that for the crisp case. The total time is $F_{\max }^{*}=45.1$ for the optimal sequence of Property 1 . We compare the result of Property 1 with that of crisp case as follows:

$$
\frac{F_{\max }^{*}-F_{\max }}{F_{\max }} \times 100=2.5 \% \text {. }
$$

\section{Conclusion}

In this paper, we have investigated the fuzzy job-shop scheduling problem with imprecise processing times. In conclusion, we point out that our work has produced the following main results for the job-shop scheduling problem with fuzzy processing times modeled as fuzzy numbers. In Section 3, we presented a fuzzy job-shop scheduling problem based on triangular fuzzy numbers. In (14), if $\Delta_{j k 2}=$ $\Delta_{j k 1}, \forall j, k$, obviously, Fig. 1 is an isosceles triangle and we obtain $t_{j k}^{*}=t_{j k}, \forall j, k$. Thus, the equations (15)-(19) will become the same as (1)-(5). If $\Delta_{j k 2}=\Delta_{j k 1}=0$, $\forall j, k$, the job-shop scheduling in the fuzzy sense of Property 1 will become the crisp job-shop scheduling of equations (1)-(5). Therefore, the job-shop scheduling in the fuzzy sense of Property 1 is an extension of the crisp scheduling of (1)-(5). 
The interpretation of Fig. 1 is as follows: When $\Delta_{j k 2}>\Delta_{j k 1}, \forall j, k$, the triangle is skewed to the right-hand side, thus obtaining $t_{j k}^{*}>t_{j k}, \forall j, k$. This means that the completion time in the fuzzy sense is longer than in the crisp case. Conversely, when $\Delta_{j k 2}<\Delta_{j k 1}, \forall j, k$, the triangle is skewed to the left-hand side, thus obtaining $t_{j k}^{*}<t_{j k}, \forall j, k$, indicating that the completion time in the fuzzy sense is shorter than the crisp case. From (14), we can see that $t_{j k}^{*}=t_{j k}+\frac{1}{4}\left(\Delta_{j k 2}-\Delta_{j k 1}\right)$ is the processing time in the fuzzy sense, where $t_{j k}^{*}$ equals the crisp processing time, $t_{j k}$, plus some variants due to the inclusion of fuzzy values.

The comparison of [10] with our work is as follows. Fortemps used six-point fuzzy numbers to represent fuzzy durations and fuzzy makespan. In his approach, the resulting fuzzy framework was not an extension of the original crisp problem. He used a simulated annealing technique to solve the fuzzy model obtaining the optimization sequence of the problem. In our approach, however, the crisp duration $x$ become an interval $\left[x-\Delta_{1}, x+\Delta_{2}\right], 0<\Delta_{1}<x, 0<\Delta_{2}$, represents an allowable range of duration. We let the fuzzy number $\tilde{x}=\left(x-\Delta_{1}, x, x+\Delta_{2} ; 1\right)$ correspond to the interval $\left[x-\Delta_{1}, x+\Delta_{2}\right]$. Then we used a signed distance method for ranking fuzzy numbers to obtain a fuzzy job-shop scheduling problem. After defuzzifying the fuzzy problem using the signed distance ranking method, we obtained a job-shop scheduling problem in the fuzzy sense. The resulting fuzzy framework in our approach is an extension of the original crisp problem. Therefore, the algorithms that were used for solving the crisp job-shop sequencing problem can also be used for solving the fuzzy problem.

\section{References}

1. Adams, J., Balas, E., and Zawack, D.: The Shifting Bottleneck Procedure for Job Shop Scheduling. International Journal of Flexible Manufacturing Systems, Vol.34, No. 3, (1987) 391-401

2. Applegate, D. and Cook, W.: A Computational Study of The Job-Shop Scheduling Problem. ORSA Journal on Computing, Vol. 3, No. 2, (1991) 149156

3. Baker, K. R.: Introduction to Sequencing and Scheduling. John Wiley \& Sons, Inc., New York (1974)

4. Bellman, R. Esogbue, A., and Nabeshima, I.: Mathematical Aspects of Scheduling and Applications. Pergamon Press, Oxford (1982)

5. Blackstone, J., Phillips D., and Hogg, G.: A State-of-The-Art Survey of Dispatching Rules for Manufacturing Job Shop Operations. International Journal of Production Research, Vol. 20, (1982) 26-45 
6. Cheng, R. Gen, M. and Tsujimura, Y.: A Tutorial Survey of Job-Shop Scheduling Problems Using Genetic Algorithms: Part I. Representation. International Journal of Computers and Industrial Engineering, Vol. 30, No. 4, (1996) 983-997

7. Croce, F., Tadei, R., and Volta, G.: A genetic algorithm for the job shop problem. Computers and Operations Research, Vol. 22, (1995) 15-24

8. Coffman, E. G. Jr.: Computer and Job-Shop Scheduling Theory. John Wiley \& Sons, Inc., New York (1976)

9. Falkenauer, E. and Bouffoix, S.: A Genetic Algorithm for Job Shop. Proceedings of the IEEE International Conference on Robotics and Automation, (1991) 824829

10. Fortemps, P.: Jobshop Scheduling with Imprecise Durations: A Fuzzy Approach. IEEE Transactions on Fuzzy Systems, Vol. 5, No. 4, (1997) 557-569

11. French, S.: Sequencing and Scheduling: An introduction to the Mathematics of the Job-Shop. John Wiley \& Sons, Inc., New York (1982).

12. Garey, M., Johnson, D., and Sethi, R.: The Complexity of Flowshop and Jobshop Scheduling. Mathematics of Operations Research, Vol. 1, (1976) 117129

13. Gen M., and Cheng, R.: Genetic Algorithms and Engineering Design, John Wiley \& Sons, Inc., New York (1997)

14. Ishii, H., Tada, M., and Masuda, T.: Two Scheduling Problems with Fuzzy Due Dates. Fuzzy Sets and Systems, Vol. 46, (1992) 339-347

15. Ishibuchi, H., Yamamoto, N., Murata, T., and Tanaka, H.: Genetic Algorithms and Neighborhood Search Algorithms for Fuzzy Flowshop Scheduling Problems. Fuzzy Sets and Systems, Vol. 67, (1994) 81-100

16. Kerr, R. M. and Slany, W.: Research issues and challenges in fuzzy scheduling. CD-Technical Report 94/68, Christian Doppler Laboratory for Expert Systems, Technical University of Vienna, Austria (1994)

17. Krishna, K., Ganeshan, K. and Janaki Ram, D.: Distributed Simulated Annealing Algorithms for Job Shop Scheduling. IEEE Transactions on Systems, Man, and Cybernetics, Vol. 25, No. 7, (1995) 1102-1109

18. McCahon, C. S. and Lee, E. S.: Fuzzy Job Sequencing for A Flow Shop. European Journal of Operational Research, Vol. 62, (1992) 294-301

19. van Laarhoven, P. J. M., Aarts, E. H. L, and Lenstra, J. K.: Job Shop Scheduling by Simulated Annealing. Operations Research, Vol. 40, No. 1, (1992) 113-125 